\title{
Time and Tense in English
}

\author{
Mary P. Harper and Eugene Chamiak \\ Brown University \\ Department of Computer Science \\ Box 1910 \\ Providence, RI 02912
}

\begin{abstract}
Tense, temporal adverbs, and temporal connectives provide information about when events described in English sentences occur. To extract this temporal information from a sentence, it must be parsed into a semantic representation which captures the meaning of tense, temporal adverbs, and temporal connectives. Representations were developed for the basic tenses, some temporal adverbs, as well as some of the temporal connectives. Five criteria were suggested for judging these representations, and based on these criteria the representations were judged.
\end{abstract}

\section{Introduction}

English sentences contain many types of temporal information. Tense is used to inform the reader (listener) of when the event associated with the main verb occurs with repect to the time of utterance. That is, tense informs the reader that an event occurs before, after, or during the time of utterance. Temporal adverbs (such as tomorrow or now) add additional information about the events in a sentence. Temporal connectives tell the reader about the temporal relationship between the events in the main clause and the events in the subordinate clause. While there is other temporal information that can be found in sentences, the following will concentrate on these three.

To extract temporal information from a sentence, it must be parsed into a semantic representation which captures the meaning of tense, temporal adverbs, and temporal connectives. A temporal representation of tense, adverbs, and temporal connectives must :

1. provide a way to reject temporally incorrect sentences, such as * "I will run yesterday."

2. allow one to reason about the temporal relationship between events. For instance, the sentence "I had run when he arrived" implies that the run event occurs before the arrival, whereas in the sentence "I was running when he arrived," the arrival and run events overlap.

3. allow the exact time of event to be unfixed until it is pinpointed based on contextual information or adverbial modification.

4. allow reference to points and intervals of time (eg precisely at 3 PM VS. for 5 hours).

This work has been supported in part by the National Science Foundation under grants IST 8416034 and IST 8515005 , and Office of Naval Research under grant N00014-79-C-0529.
5. allow parsing of temporal information in sentences to be simple and compositional.

These criteria were used to judge previous temporal representation research (Bruce (1972), Homstein (1977, 1981), Yip (1985)). None fulfilled all five criteria. The criteria will also be used to judge the representations developed here.

\section{Tense}

The representations for tense, adverbs, and temporal connectives developed here is based on McDermott's (1982) temporal logic. McDermott's "point-based" temporal logic was chosen because it is not unusual to talk about the beginning and end points of a period of time or an event. In fact, the semantics of tense developed here relate the endpoints of events in sentences. This representation of tense provides a flexibility not found in many other representations of tense (eg. (Hornstein, 1977,1981)). Flexibility is important since events can extend over tense boundaries (for instance, "In 3 minutes, the boy will have run for 24 hours."). Any representation of events in time must capture the fact that some events do not always wholly occur in the past, present, or future with respect to the time of utterance.

The tense rules are compositional and require the following relations : < (before), > (after), = (cotemporaneous), $\leq$ (before or cotemporaneous), and $\geq$ (after or cotemporaneous). It is assumed that events are "unit" events and have a beginning and an end point, where the beginning of an event is before or simultaneous to its end point. The endpoint of an event need not imply the achievement of the purpose with which the event was initiated (eg. the existence of the end point of a winning event need not imply that the state of having won is achieved). To capture the meaning of simple as well as more complex tenses, we introduce the following events :

1. Utterance Event - This is simply the speaking event associated with a sentence.

2. Main Event - This is the event indicated by the main verb of the sentence. For instance, the run event in the following sentence is the main event: "I have been running to the store."

3. Perfect Eyent - This is the time interval referred to in sentences like: "Bill had eaten at 3 PM," which describes an eat event in the "distant past." This sentence implies the existence of an event or time interval which occurs after the main event (eat) but before the utterance event. 
4. Progressive Event - This is the time interval from which the main event extends into the past and into the future. The progressive event may have no correlation with a "real world" event, but its existence predicts certain phenomena in our model of temporal adverbs and connectives. It can be thought of as a place holder, or the minimal possible duration of a main event with progressive aspect.

The following five rules describe the semantics of tense both in English and in our representation. The verbs in a sentence are parsed left to right (assuming an ATN, which is the parser in which these tense rules were implemented). One of the following three rules is triggered by the tense of the first verb in the sentence. "Event" (in the first three rules) can be a main event, a perfect event, or a progressive event depending on the sentence.

1. Past rule : This rule implies that there exists some event that must end before the beginning of the utterance event.

( $<$ (end event) (begin utterance-event))

2. Present rule: This rule implies that there exists some event that is either cotemporaneous with the utterance event or can begin at or after the beginning of the utterance event. Which is asserted seems to depend on the aspect of the verb associated with event.

If the current verb is stative then

(and (= (begin event) (begin uterance-event)) ( $=$ (end event) (end uterance-event))

If the current verb is not a stative then ( 2 (begin event) (begin uterance-event))

3. Euture rule : This rule implies that there exists some event that must begin after the end of the utterance event.

( $>$ (begin event) (end utterance-event))

The following rules are required to interpret the more complicated perfect and progressive tenses.

4. Perfect rule : This rule is triggered by the word have followed by a past participle. The event in the rule can be a progressive or a main event. ( $\leq$ (end event) (begin perfect-event))

5. Progressive rule : This rule is triggered by the word be followed by a progressive verb form. The event in the rule can only be a main event.

(and ( $\leq$ (begin main-event) (begin progressive-event)) ( $Z$ (end main-event) (end progressive-event)))

These rules combine in a compositional way to define the more complicated tenses. For instance the past perfect progressive tense combines the past rule with the perfect and progressive rules. Thus the sentence "Jack had been running" is represented as follows :

(and (inst utterance6 utterance-event)

(< (end have2) (begin utterance6)) ; past rule

(inst have2 perfect-event)

( $<=($ end be3) (begin have2)) ; perfect rule

(inst be 3 progressive-event)

(inst run64 run)

$(<=$ (begin run64) (begin be3)) ; progressive rule

( $>=$ (end run64) (end be 3 ))

(inst run64 main-event)

(name Jack16 Jack)

$(:=$ '(agent run64) Jack16))
A "temporal" picture can be drawn for this sentence (see Figure 1). Note that the picture is only one possible depiction of the actual meaning of this representation.

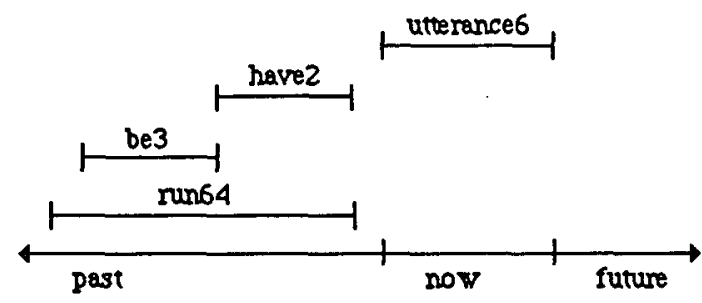

Figure 1. "Jack had been running."

A parser uses the semantic rules of tense as follows. After checking the tense of the first verb, the parser checks to see if the verb is the word will. If it is, then move to the next verb and mark the event associated with this verb as a future event. Assert either the past, present or future rule depending on the tense associated with the "event" of the current verb. Now check to see if the current verb is have followed by a past participle. If so, then assert the perfect rule relating the perfect event (the event associated with have) and the event associated with the verb to the right of have, and move to that verb. After checking for perfect tense, the parser looks for a form of the word be followed by the progressive form of a verb. This signals the progressive rule, which relates the progressive event with the main event.

The representation adopted has some support in linguistic literature, and there are some similarities to the representations developed by Bruce (1972), Hornstein (1977, 1981), Reichenbach (1947), and Yip (1985), although there are many differences. One difference between this representation and previous representations of tense is how present tense is defined. All past theorists have considered present tense as indicating that the main event is cotemporaneous with the time of utterance. However, aspect of verb seems to affect the meaning of present tense. In present tense sentences, there exists a curious phenomenon which can best be understood by examining the following two sentences:

1. I leave at eight o'clock tomorrow.

2. *I have a dog tomorrow.

Aspect interacts with present tense requiring a more complicated present rule in a theory of tense.

\section{Adverbials}

The representation of several types of temporal adverbs will be considered, as well as how the meaning of these adverbs combines with the meaning of the tense. As in the representation of tense, we require the following relations : $<,>, \leq, z$, and $=$. We will consider how to predict incorrect combinations of tense and adverbs based on the representations of tense and adverbs developed here.

As suggested by Hornstein (1977), we adopt the idea that which event is modified by an adverbial is an important issue (since we introduce multiple events in our 
definition of some of the basic tenses). The ambiguity concerning which event is modified can best be seen in the following example: "I had eaten at 3." This sentence has a utterance event, which can not be directly modified by an adverb. It can be modified by context, and it can be modified when some event which is cotemporaneous to the utterance event is modified. The past perfect sentence introduces a perfect event and a main event (eat) in addition to the utterance event. If we assume that the main event is modified, then the time of "eating" must overlap 3 o'clock. If it modfies the perfect event, then by the time 3 o'clock came around the "eating" was complete. In general, we adopt the idea that which event is modified is ambiguous, and thus a disjunction of possibilities is asserted.

Since Hornstein (1977) and Yip (1985) examined the three adverbials tomorrow, yesterday, and now, we will concentrate on these three. Each of these adverbs shares the fact that they are defined with respect to the time of the utterance event (today is also included in this category of adverbs though not discussed here). The representations of now, tomorrow, and yesterday follow:

Now : Now is defined to be a time interval which is cotemporaneous with the utterance event. Thus, the representation of some specific now is :

(and (inst now 16 time-interval) (= (begin now 16) (begin utterance2)) (= (end now16) (end utterance2)))

Tomorrow : Tomorrow is also defined with respect to the time of utterance. Notice that the duration of tomorrow is precisely 24 hours (as indicated in the fourth conjunct).

(and (inst tomorrow3 day)

(> (begin tomorow3) (end utterance2))

(s (begin tomorrow3)

(+ (end utterance 2$)(* 24$ hour $))$

$(=(-($ end tomorrow 3$)$ (begin tomorrow 3$))$

(*24 hour)))

Yesterday : Yesterday is defined with respect to the time of utterance, and has a 24 hour duration. (and (inst yesterday 3 day)

(< (end yesterday 3 ) (begin utterance2))

$(\geq$ (End yesterday 3 )

(- (begin utterance2) $(* 24$ hour $))$ )

(= (- (end yesterday 3$)$ (begin yesterday 3$))$

(*24 hour)))

To satisfy criterion 1 , this model should be able to predict temporal inconsistencies between temporal adverbs and tense. Any event in a sentence can be modified by an adverb if the event can potentially overlap the period of time associated with the adverb. Thus we introduce the overlap rule of adverb-tense agreement :

Overlap Rule : An event can be modified by a temporal adverb iff the time period associated with an adverb can overlap the time period associated with the event without some temporal contradiction. That is, if the following assertion does not contradict other temporal assertions associated with the sentence, then the events can overlap : (and ( $\leq$ (begin event) (end adverb))

( $\geq$ (end event) (begin adverb)))

Because events are defined flexibly in this tense representation, some events can cross tense boundaries.
For correct adverb-tense agreement, the events in the sentence must be "anchored" to the event associated with the first verb in the sentence, that is the event that determines the tense of the sentence (note that will has no event associated with it). The need for this anchoring can best be shown with following examples :

*Now, he will have eaten. (excluding modal reading)

* Yesterday, he will have eaten. (excluding modal reading)

Tomorrow, he will have eaten.

The tense stucture of each of these sentences (as given by our tense rules) introduces three events, an utterance event, a perfect event, and a main event. Notice that the only event that is necessarily in the future is the perfect event. The main event could overlap yesterday or now, as well as tomorrow. Thus it would seem that given that the main event can be modified by yesterday or now, the first two sentences should be correct. However, except for possible modal readings, these sentences are not acceptable. We account for this with the following rule:

Anchoring rule : If the time period of the event associated with the first verb of a sentence can overlap the time period associated with an adverb, then the adverb can modify that event and can potentially modify the other events in the sentence (based on the overlap rule). The utterance event can not be modified using the anchoring rule.

To show how these two rules (anchoring and overlap) are used, examine the sentence: "He is running now."

Step 1 : Get the basic representations of the adverbial and the tense.

(and (inst utterance6 utterance-event)

; adverb representation

(inst now5 time-interval)

(= (begin now5) (begin utterance6))

(= (end now5) (end utterance6))

; tense representation

(inst bel progressive-event)

(= (begin be1) (begin utterance6))

( $=$ (end be 1 ) (end utterance6))

(inst run4 run)

(inst run4 main-event)

( $\leq$ (begin run4) (begin be 1$)$ )

( $\geq$ (end run 4 ) (end be 1$)$ ))

Step 2 : Check to see if the anchor event can overlap the adverb. Assume that $C H E C K$ is a function that returns true if the overlap is possible. Since Bel and Now5 occur at the same time, the result of the test is true.

(CHECK (and ( $\leq$ (begin be 1) (end now5))

( $Z$ (end be 1$)$ (begin now5))))

Step 3 : If the overlap check of the anchor returns true, then do overlap checks on the remaining events. For those that return true, assert a disjunction of ways that the adverb can modify the events. In this case assert :

$$
\begin{aligned}
& \text { (or (and }(\leq \text { (begin be } 1)(\text { end now5)) } \\
&((\text { end be } 1)(\text { begin now5))) } \\
& \text { (and }(\leq \text { (begin run4) (end now5)) } \\
&(\geq \text { (end run4) (begin now5))) }
\end{aligned}
$$

An example of a sentence in which the anchor event and the adverb can not overlap is *"He ran tomorrow." The 
Table 1. Tense-Adverb Compatibility

\begin{tabular}{|c|c|c|c|}
\hline \multirow{3}{*}{$\begin{array}{r}\text { Past } \\
\text { Past Progressive }\end{array}$} & Yesterday & \multirow{2}{*}{$\frac{\text { Now }}{\text { error }}$} & Tomonow \\
\hline & ok & & error \\
\hline & ok or Prog. Rule* & Prog. Rule only & Prog. Rule only \\
\hline Past Perfect & ok & error & error \\
\hline Present & error & ok & ok or error \\
\hline Present Progressive & error & ok & Prog. Rule only \\
\hline Present Perfect & error & ok & enror \\
\hline Future & error & error & ok \\
\hline Future Progressive & error & enror & ok or Prog. Rule \\
\hline Future Perfect & error & enror & ok \\
\hline
\end{tabular}

* Reference to "Prog. Rule" refers to a modification of the Past Progressive Rule suggested by Homstein (1977), which is ignored in this paper.

run event can not overlap tomorrow (because the run event ends in the past and tomorrow begins in the future), and the sentence is therefore reported as erroneous. See Table 1 for the adverb-tense predictions of our model. Modal readings are ignored in this paper.

There are other adverbials which are interpreted relative to the time of utterance (for instance, this week, next week, and last year). It is not difficult to imagine how to represent these adverbials. There are also some adverbials which need not be defined relative to the time of utterance. These include all of the clock calendar adverbials, such as Sunday and midnight. For example the representation of a specific Sunday is:

(and (inst sunday 3 day)

$$
(=(-(\text { end sunday } 3) \text { (begin sunday } 3))
$$

$$
\text { (*24 hour))) }
$$

Sunday 3 can not be placed in the past, present, or future. However, when Sunday is used in a sentence, we can determine whether we mean a past, present, or future Sunday. Durational adverbials can also be easily represented (somewhat like the definition of Sunday).

There are other adverbials which like clock calendar adverbials are not interpretted with respect to the time of speech. One such temporal adverb is just. This adverb is distinguished from the word just, meaning only. To see how it is used, examine the following sentences :

1. I just ate lunch.

2. I was just eating lunch.

3. I had just eaten lunch.

4. * I just eat.

5. I am just eating lunch.

6. I have just eaten lunch.

7. * I will just eat lunch.

8. I will be just eating lunch.

9. I will have just eaten lunch.

Notice that just can not be used in simple present or simple future tense. This adverb requires the existence of some event in the sentence that begins immediately after the start of the event modified by just. Sentences 5 and 8 require progressive events to represent their tense structure. This tense representation allows our model to predict the correctness of these two sentences. The definition of just follows :

Just : Just relates two events, where Ev1 can be the main event, the progressive-event, or the perfect-event, and Ev2 can be the utterance-event, the perfect-event, or the progressive-event. Ev1 and Ev2 must not be separated by another event introduced by the sentence. $\partial$ is some small value which is determined by context.

$$
\begin{aligned}
& (<\text { (begin Ev1) (begin Ev2)) } \\
& \text { if }(\leq(\text { End Ev1) (begin Ev2)) then } \\
& \text { assert }(\leq(- \text { (begin Ev2) (End Ev1)) } \partial) \\
& \text { else } \\
& \quad \text { assert }(\leq(- \text { (begin Ev2) (begin Ev1)) } \partial)
\end{aligned}
$$

There are many other temporal adverbials that need to be represented, among them recently, afterwards, earlier, lately, already, and soon. Most of these relate two events, in much the same way as temporal connectives which will be our next topic.

\section{Temporal Connectives}

A few issues must be examined before we present our representation of temporal connectives. First it should be pointed out that temporal connectives are subordinators. Most subordinators do not restrict the tense of the subordinate clause given the tense of the main clause. The tense of the main clause does restrict the tense of the subordinate clause when the subordinator is a temporal connective. The following results are predicted by Homstein (1977) :

John left when Harry
1. *arrives.
4. arrived
7. *will come.
2. *is arriving.
5. was arriving. 8 . *will be coming.
3. *has arrived.
6. had come.
9. * will have arived.

By studying the above example, one might suggest that the tense of the main clause and the tense of the subordinate clause must have the same tense (disregarding progressive and perfect aspects). This seems to be true for all past and present tenses. There are some restrictions of this statement, however, since the will/shall construction of future tense is not allowed in temporal subordinate clauses. As pointed out by Leech (1971):

"In dependent clauses introduced by conditional and temporal conjunctions if, unless, when, as soon as, as, etc., the future is denoted by the ordinary Present Tense instead of the construction with will/shall:

I'll tell you if it hurts.

When the spring comes, the swallows will return.

Jeeves will announce the guests as they arrive." (p.59) 
If the will/shall construction is used in a subordinate clause introduced by a temporal connective, then the reading of the sentence is not a future but a modal reading. This fact was not noticed by Hornstein $(1977,1981)$ or Yip (1985). Hornstein allows both present tense and will/shall future tense to occur in temporal subordinate clauses. Yip only allows the will/shall future tense to occur in the subordinate clause ${ }^{1}$.

Rather than include the syntactic needs of temporal connectives in our semantic representation, it seems wiser to include the requirement at a syntactic level. That is the tense of the first verb of the main clause restricts the tense of the first verb in the temporal subordinate clause. If the tense of the first verb in the main clause of the sentence is past or present, then the tense of the first verb in the subordinate clause must have like tense. If the tense of the first verb in the main clause is future tense, then the tense of the first verb in the subordinate clause must be present tense (though it will be semantically interpretted as future tense).

Now, we must consider how to extract the temporal meaning of sentences of the form sentence-temporal connective-sentence. Each clause will be given a temporal representation as indicated in the tense representation section of this paper. Both clauses will have the same time of utterance, since an utterance event is created only for a sentence. The only subtlety is the requirement that present tense in a subordinate clause be interpretted using future semantics when the main clause has future tense. After each clause is represented, the semantics for the temporal connective must be invoked. Each temporal connective requires its own definition, as pointed out by Hornstein(1977). These definitions will determine the temporal relationship between the events in the main clause and the events in the subordinate clause. We will present the definitions for five temporal connectives: when, while, until, before, and after. Because these definitions can use the representation of tense associated with each clause in a sentence to interrelate the events between clauses, the strength of the tense representation is increased.

When : align the anchor events to determine the relationship between events of the clauses. If the main events of both clauses are the anchor events, then the events may occur at exactly the same time, though not necessarily.

(and (= (begin anchor-event(main-clause)) (begin anchor-event(subordinate-clause)))

( $=$ (end anchor-event(main-clause))

(end anchor-event(subordinate-clause))))

While : align the anchor and main events of the clauses. Check to see if the alignment of both is possible. If check returns faise then reject the sentence.

1. Yip(1985) and Hornstein(1977) try to deal with this temporal connective phenomenon and adverb-tense agreement with a unified theory. Hornstein's theory accepts sentences of the form *"I have eaten tomorrow" so that the sentence "I will leave when he has eaten" is acceptable. Yip modifies Hornstein's theory to get rid of the yesterday-present perfect error, but the modification does not allow a future tense main clause to have a present tense subordinate clause.

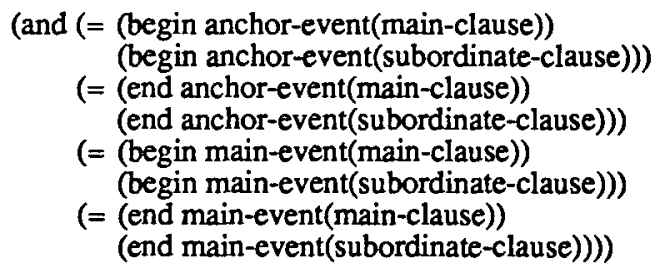

Until : requires in most cases that the main event of the main clause end when the the main event of the subordinate clause begins. If the tense representation of the subordinate clause has a perfect event and no progressive event, then the main event of the main clause must end when the main event of the subordinate clause ends.

If subordinate clause has a perfect but no progressive event

(= (end main-event(main-clause)) Else (end main-event(subordinate-clause)))

(= (end main-event(main-clause)) (begin main-event(subordinate-clause)))

Before : requires that the anchor event of the main clause end before the beginning of the main event of the subordinate clause.

( $<$ (end anchor-event(main-clause))

(begin main-event(subordinate-clause)))

After : requires in most cases that the main event of the main clause begin after the end of the anchor event of the subordinate clause. If the main clause has a progressive event, then the anchor event of the main clause begins after the end of the anchor event of the subordinate clause and the main event of the subordinate clause ends before the end of the main event of the main clause.

If main clause has a progressive event then

(and (< (end anchor-event(subordinate-clause)) (begin anchor-event(main-clause)))

( $<$ (end main-event (subordinate-clause))

Else (end main-event (main-clause))))

( $<$ (end anchor-event(subordinate-clause)) (begin main-event(main-clause)))

Notice that before and after are not always inverses of one another. Consider the following two sentences :

1. I ate before he was running

2. He was running after I ate.

If before and after were inverses, then sentence 1 and 2 would have equivalent meanings which they do not. The definitions of before and after capture this assymetry.

Two Examples are presented to acquaint the reader with the representation of sentences joined by temporal connectives. The first is : "Mary ate when Joe was eating."

I. Represent the clauses.

(and (inst utterance3 utterance-event)
; "Mary ate"
(inst eat22 eat)
(inst eat22 main-event)
(< (end eat22) (begin utterance3))
(name Mary22 Mary)
(:= (agent eat22) Mary22)




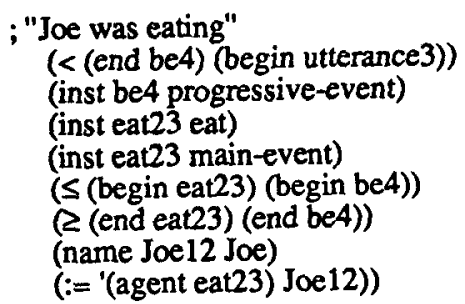

II. Do semantics for when.

Note that the anchor event for the main clause is eat 22 , and the anchor event for the subordinate clause is be4.

(and ( = (begin eat22) (begin be4)) (= (end eat22) (end be4)))

This sentence can depicted as follows (see Figure 2):

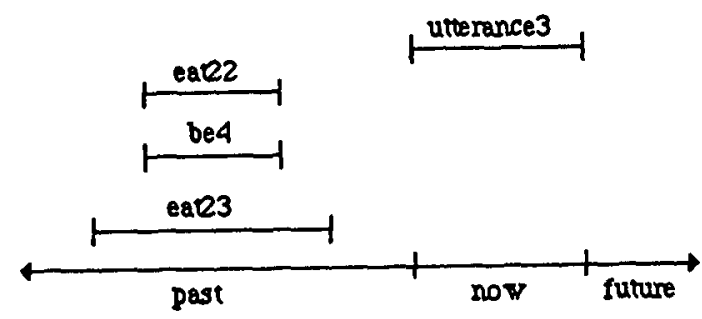

Figure 2. "Mary ate when Joe was eating."

This implies that eat 23 can begin before and end after eat 22 , though they could be exactly coincident. This seems to be the desired interpretation of this sentence. This is not the meaning that Hornstein's model would give this sentence. Yip (1985) introduces progressive aspect rules to Hornstein's tense rules to get exactly this result.

The second example consists of an analysis of the sentence : "Mary ate when he had eaten."

I. Represent the clauses.

(and (inst utterance 3 utterance-event)

; "Mary ate" representation

(inst eat22 eat)

(inst eat22 main-event)

(< (end eat22) (begin utterance 3 ))

(name Mary22 Mary)

(:= '(agent eat22) Mary22)

; "He had eaten" representation

(< (end have3) (begin utterance 3$)$ )

(inst have 3 perfect-event)

(inst eat23 eat)

(inst eat 23 main-event)

( $\leq$ (end eat23) (begin have3))

(inst Jack 12 Jack)

(:= '(agent eat23) Jack12))

II. Do semantics for when.

Note that the anchor event for the main clause is eat22, and the anchor event for the subordinate clause is have3.

(and $(=($ begin eat 22$)$ (begin have 3$)$ ) ( $=$ (end eat 22 ) (end have3)))
This sentence can be depicted as shown in Figure 3. Thus, it can be seen that eat 23 must end by the beginning of eat22. This seems to be the correct interpretation of this sentence, and was exactly the interpretation that Homstein's when rule makes. These two examples show that the when rule predicts very different relationships between events depending on the tenses in the clauses.

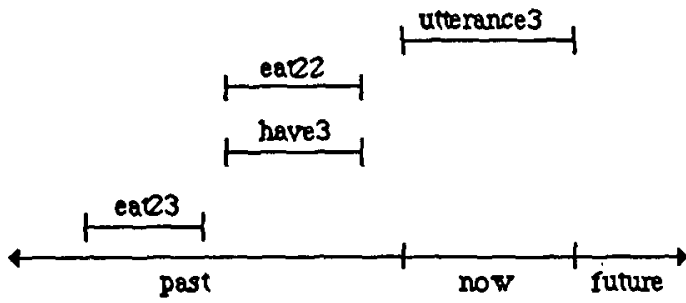

Figure 3. "Mary ate when Jack had eaten "

\section{Conclusion}

This paper describes a preliminary study of the temporal phenomena found in English sentences. Many issues have been ignored for simplicity. For instance, the issue of habitual readings of verbs was not examined. The meanings of verbs with temporal aspects (such as plan) were also not considered. In addition, we did not consider how to relate (in time) events from different sentences. The only events from different sentences that can be related are the utterance events. If two sentences occur in sequence, one can conclude only that the utterance event of the first ends before the utterance event of the second.

The model developed here can, however, temporally order events within a sentence. Five criteria were suggested at the beginning of the paper for the representation of temporal information found in an English sentence. These criteria guided the development of our model. All criteria were met, except the compositional parse criterion in a few cases. There seem to be unavoidable special cases which can not be captured in compositional tense, adverb, and temporal connective rules. For instance, the meanings of some adverbs require tense information to determine their correct representations (e.g. just).

\section{References}

Allen, James. Maintaining Knowledge About Temporal Intervals. CACM, 1983, 26, 832-843.

Bruce, Bertram C. A Model for Temporal References and Its Application in a Question Answering Program. Artificial Intelligence, 1972, 3, 1-25.

Charniak, E., Gavin, M., and Hendler, J. The Frail/Nasl Reference Manual. Brown University Technical Report CS-83-06, 1983.

Charniak, E. and McDermott, D. Introduction to Artificial Intelligence. Reading, MA : Addison-Wesley Publishing Company, 1985. 
Hornstein, Norbert. Towards a Theory of Tense. Linguistic Inquiry, 1977, 8, 521-557.

Hornstein, Norbert. The Study of Meaning in Natural Language, In N. Hornstein \& D. Lightfoot (Eds.), Explanation in Linguistics. New York : Longman, 1981.

Leech, Geoffrey N. Meaning and the English Verb. London : Longman, 1971.

McDermott, Drew. A Temporal Logic For Reasoning About Processes And Plans. Cognitive Science, 1982, 6, 101-155.

Reichenbach, Hans. Elements of Symbolic Logic. New York : MacMillan, 1947.

Yip, Kenneth M. Tense, Aspect and the Cognitive Representation of Time. IJCAI Proceedings, 1985, 806-814. 